\title{
An Ellipsometrical and AFM Study of Ferritin Adsorption Kinetics on a Gold Surface
}

\author{
L. Vladimirova ${ }^{a, *}$, A. Andreeva ${ }^{b}, \mathrm{~V} \cdot \mathrm{Savov}^{a}, \mathrm{~A} \cdot \mathrm{GritzKow}^{c}$ And M. Burova $^{b}$ \\ ${ }^{a}$ Faculty of Physics, Department of Atomic Physics, Sofia University "St. Kliment Ohridski" \\ 5 James Boucher Blvd., 1164 Sofia, Bulgaria \\ ${ }^{b}$ Faculty of Physics, Department of Semiconductors, Sofia University "St. Kliment Ohridski" \\ 5 James Boucher Blvd., 1164 Sofia, Bulgaria \\ ${ }^{c}$ Faculty of Physics, Department of Optics, Sofia University "St. Kliment Ohridski" \\ 5 James Boucher Blvd., 1164 Sofia, Bulgaria
}

\begin{abstract}
The kinetics of ferritin adsorption on gold $(\mathrm{Au})$ surface was studied with ellipsometry and atomic force microscopy. The thickness and the refraction index of the adsorbed layer were determined using a theoretical model system: air-interface layer-solution-adsorbed layer-gold surface. The relations between the parameters of the adsorbed layer and $\mathrm{pH}$ and the protein concentration are obtained. It seems that the ferritin adsorption is a relatively slow multi-stage process. The obtained results enable modelling of the protein adsorption on blood vessels and tissues in pathological processes. The kinetics of protein adsorption could be implemented in medical applications and in development of new biosensor systems.
\end{abstract}

PACS numbers: 79.60.Dp, 42.62.Be, 73.30. $+\mathrm{y}, 78.68 .+\mathrm{m}$

\section{Introduction}

The interaction between proteins and surfaces is of great interest to biological practice and technology [1]. The investigation of such phenomena as adsorption, critical nuclei formation and film growth gives us key understanding of important processes in biomaterials, biosensors, biotechnology and nanotechnology [2].

Experimental and theoretical works are devoted to the kinetics of adsorption in simple model systems [3-8], assuming that the protein molecules repeatedly impinge upon the surface, before they adsorb on it [9]. According to this model, the molecules do many attempts to overcome the surface barrier before they stabilize on the surface and they do not further desorb into the solution $[10,11]$.

The goal of this work is to study ellipsometrically and by atomic force microscopy the adsorption kinetics of proteins and the dependence of surface protein concentration on $\mathrm{pH}$ and protein concentration in solutions, as well as the influence of the interface layer formed at the air-solution surface on the measured parameters. The ferritin was used as a model system because it plays a key role in the biochemical transformations of iron and oxygen - processes of extraordinary significance for organism existence.

* corresponding author; e-mail: vladimirova@phys.uni-sofia.bg

\section{Materials and methods}

\subsection{Materials}

Objects - horse spleen ferritin (HoSF) (Type I, Sigma). The following buffer solutions are used: $(0.05 \mathrm{M})$ $\mathrm{KH}_{2} \mathrm{PO}_{4} / \mathrm{NaHPO}_{4}, \mathrm{pH} 7.0 / 25^{\circ} \mathrm{C}$ and $(0.05 \mathrm{M}) \mathrm{NaHCO}_{3}$, $\mathrm{pH} 10.0 / 25^{\circ} \mathrm{C}$. All chemicals applied for the preparation of the buffer solutions are of chemical grade from HANNA Instrument.

The following standard cleaning procedure in ultrasonic bath at room temperature is applied to remove organic pollutions from the glass substrate: (a) washing in tetrachloromethane, then (b) in acetone, and finally (c) in ethanol. The full process duration is ca. $15 \mathrm{~min}$. All the solvents are of electronic grade.

The $50 \mathrm{~nm}$ thick gold ( $\mathrm{Au}, \mathrm{N} 9999)$ layer is deposited onto the substrate immediately after cleaning by thermal evaporation in a vacuum chamber with $<10^{-4} \mathrm{~Pa}$ working pressure. The diameter of the gold surface is $12 \mathrm{~mm}$. It is washed in piranha solution $\left(\mathrm{H}_{2} \mathrm{SO}_{4}: \mathrm{H}_{2} \mathrm{O}_{2}=3: 1\right.$, $\mathrm{v} / \mathrm{v}$ ) for $1 \mathrm{~min}$, then washed with water and ethanol and measured to ensure that the surface is thoroughly cleaned.

The data are processed and fit using ellipsometrical softwear (ELLI3M) and Origin7.

\subsection{Ellipsometric measurements}

The measurements are carried out at room temperature using a null ellipsometer (LEPh-3M-1, Russia) 
at $\lambda_{0}=632.8 \mathrm{~nm}$. The angle of incidence on the air-solution boundary is $\phi=45^{\circ}$ which corresponds to $\phi^{\prime}=32^{\circ}$ on the gold surface. All the ellipsometric measurements are made in situ, as the substrate is placed into a Petri dish filled with the corresponding solution (ca. $6 \mathrm{ml}$ ). The uncertainties for the angle of incidence setting and the positioning of the polarizer, compensator and analyzer do not exceed $0.2 \mathrm{mrad}$. It is important to mention that each ellipsometrical measurement takes about $300 \mathrm{~s}$, which is negligible in comparison to the duration of a monolayer film growth.

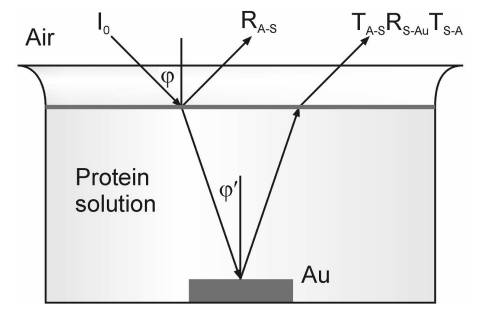

Fig. 1. Schematic allignment for ellipsometric measurements of the "air-solution-gold surface" system. $I_{0}$ - incident beam, $R_{\mathrm{A}-\mathrm{S}}$ - reflected one from airsolution boundary, and $T_{\mathrm{A}-\mathrm{S}} R_{\mathrm{S}-\mathrm{Au}} T_{\mathrm{S}-\mathrm{A}}-$ a beam reflected from gold substrate; $\phi$ and $\phi^{\prime}$ - angles of incidence onto solution and gold surfaces.

Applying these experimentally determined angles, $\Psi$ and $\Delta$, one can get information about the structure of the investigated system by solving the inverse problem.

The distance between the air-solution boundary and the gold surface is $c a .2 \mathrm{~mm}$, i.e. the both reflected beams, $R_{\mathrm{A}-\mathrm{S}}$ and $T_{\mathrm{A}-\mathrm{S}} R_{\mathrm{S}-\mathrm{Au}} T_{\mathrm{S}-\mathrm{A}}$, are separated at about $1.8 \mathrm{~mm}$ (Fig. 1). This distance is large enough to measure the ellipsometrical angles for both beams independently, in one experiment. The auxiliary study of the air-solution boundary is necessary because the properties of the condensed matter at the boundary sometimes differ from those into the volume even for pure liquids [12] and thus may influence the ellipsometrical angles for $T_{\mathrm{A}-\mathrm{S}} R_{\mathrm{S}-\mathrm{Au}} T_{\mathrm{S}-\mathrm{A}}$ beam. This influence can be compensated by introducing an interface layer in the physical model, at the boundary between the protein solution and the air. It is further assumed that this layer is homogeneous, isotropic and thin. Consequently, the ellipsometrical model for $R_{\mathrm{A}-\mathrm{S}}$ beam comprises a very thin layer with unknown optical thickness $t_{\mathrm{F}}=n_{\mathrm{F}} d_{\mathrm{F}} \ll \lambda_{0}$ and refractive index $n_{\mathrm{F}}$, embraced by two isotropic homogeneous semi-infinite media - air $\left(n_{\mathrm{A}}=1.000\right)$ and protein solution $\left(n_{\mathrm{S}}\right)$. $n_{\mathrm{S}}$ depends on the protein concentration, the protein chemical structure and the acidity of the solution [13]. To maintain the $\mathrm{pH}$ of the protein solutions constant, two buffers are used as solvents.

They and the parameters of the interface layer $\left(n_{\mathrm{F}}\right.$ and $t_{\mathrm{F}}$ ) define the amplitude reflection coefficients $r_{\mathrm{S}}$ and $r_{\mathrm{P}}$ for $R_{\mathrm{A}-\mathrm{S}}$ beam and correspondingly - the ellipsometrical angles. This allows the calculation of the forward and backward amplitude transmission coeffi- cients of the interface layer, $t_{1 \mathrm{~S}}, t_{1 \mathrm{P}}$ and $t_{2 \mathrm{~S}}, t_{2 \mathrm{P}}$, for $T_{\mathrm{A}-\mathrm{S}} R_{\mathrm{S}-\mathrm{Au}} T_{\mathrm{S}-\mathrm{A}}$ beam. Using these coefficients and the ellipsometrical angles for $T_{\mathrm{A}-\mathrm{S}} R_{\mathrm{S}-\mathrm{Au}} T_{\mathrm{S}-\mathrm{A}}$ beam, one can calculate the amplitude reflection coefficients $\check{r}_{\mathrm{S}}$ and $\check{r}_{\mathrm{P}}$ for the reflection from the protein layer-gold substrate system and finally, to determine the parameters of a protein film adsorbed on the gold surface.

It is assumed that the concentration of the solution is nearly constant during the whole experimental time. Indeed, using the specific gravity of $1.45 \mathrm{~g} / \mathrm{cc}$ for ferritin in solid condition and its characteristic diameter of $12 \mathrm{~nm}$ [14] one can estimate that even adsorption of several monolayers in dense hexagonal packing onto gold substrate with area $c a .1 \mathrm{~cm}^{2}$ leads to maximum change in the average solution concentration less than $5 \%$.

\subsection{Atomic force microscopy measurements}

The absorbed layer is studied contactless using atomic force microscopy (AFM). The ferritin is adsorbed onto a gold substrate. The gold substrate is immersed in the cuvette with the studied solution. The protein is absorbing during $180 \mathrm{~min}$. Then the substrate is removed and put to dry in horizontal position without additional drying. The measurements are taken by atomic force microscope Anfatec Instruments AG, Germany. The dynamic non-contact regime is used. Maximal scanning region is $30 \mu \mathrm{m}$.

\section{Results and discussion}

The adsorption of proteins on the gold surface can be influenced by different factors, the most important of which are the protein concentration, temperature, $\mathrm{pH}$ of the solution as well as the total protein amount in the solution (it is necessary to be much greater than the adsorbed protein mass in order to be maintained steady concentration). These factors may impact not only on the adsorption kinetics (time dependence of the layer thickness, etc.) but also on the optical parameters of adsorption (refractive index) because of their effect on the protein structure.

The calculation of the adsorbed layer thickness from the ellipsometric data reveals that the adsorbed layer thickness grows with almost constant rate (Fig. 2). The surface concentration $\Gamma\left(\mathrm{ng} / \mathrm{mm}^{2}\right)$ has been analysed according to Stenberg et al. [15].

It is worthwhile to notice the fact that at low ferritin concentration $(0.01 \mathrm{mg} / \mathrm{ml})$ in neutral solution $(\mathrm{pH} 7.0)$ this rate is sixfold lower than at higher concentration or in alkaline solutions. A possible explanation of this fact is the structural protein change in the nearest to the gold substrate sub-layers caused by the Coulomb forces between the ferritin molecules and the charged substrate.

AFM images of adsorbed ferritin measurements are showed in Fig. 3. The results confirmed those results received by ellipsometry. There are dendrite formations to observe. The possible mechanism for their forming is comprised of the following stages: fast adsorption of the 

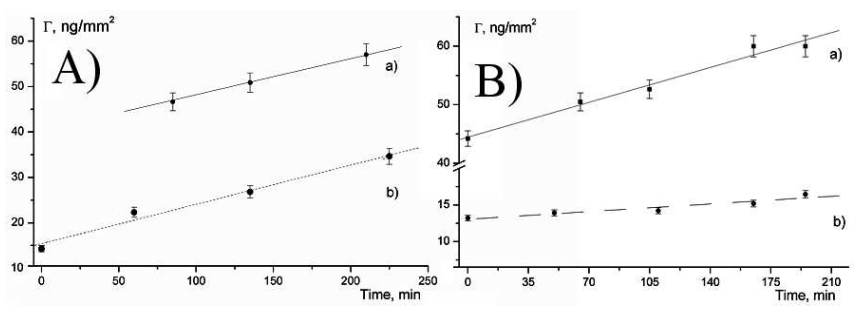

Fig. 2. Kinetics of ferritin adsorption at (A) $\mathrm{pH} 7.0$ and (B) $\mathrm{pH} 10.0$ for two different solution concentrations: (a) $0.1 \mathrm{mg} / \mathrm{ml}$ and (b) $0.01 \mathrm{mg} / \mathrm{ml}$.

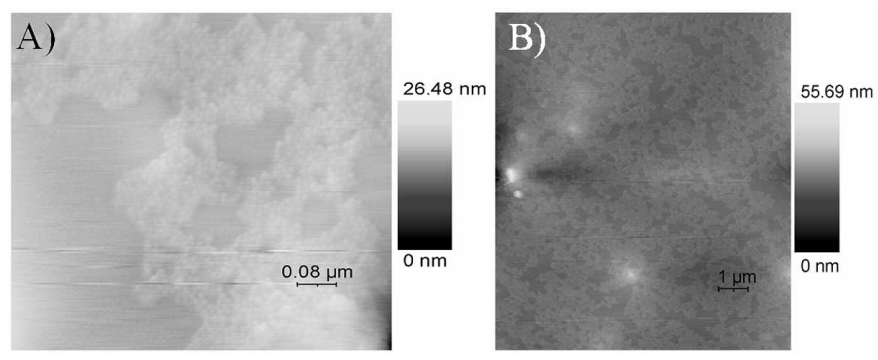

Fig. 3. AFM images of ferritin adsorption $(0.1 \mathrm{mg} / \mathrm{ml})$ in buffer $\mathrm{pH} 7.0$ used dynamic non-contact regime: (A) after $10 \mathrm{~min},(\mathrm{~B})$ after $120 \mathrm{~min}$.

ferritin molecules at the active sites on gold surface; slow growth of linear segments as they simultaneously grow in width; the change of the orientation of a segment. During this reorientation its structure transforms into more shallow and wider formation.

\section{Conclusions}

The thickness of the adsorbed layer changes for ferritin at nearly constant rate, probably not including the earlier stage of growth, as the steady state of the adsorption process is practically not achieved. The rate of layer growth is slightly influenced by $\mathrm{pH}$ and the solution concentration, probably due to alternations in the protein structure.
Most likely this is associated with the specific structure of the protein as well as with its physiological functions leading to the development of different pathological conditions, i.e. the adsorption of the studied protein depends on its structure and therefore, on the specific roles which it plays in the organism. The considered interaction can be discussed as one of the causes for different pathological processes, such as plaque building and precondition of hearth diseases $[16,17]$.

\section{References}

[1] E. Topoglidi, A.E.G. Cass, G. Gilardi, S. Sadeghi, N. Beaumont, J.R. Durrant, Analyt. Chem. 70, 5111 (1998).

[2] R.M. Nyquist, A.S. Eberhardt, L.A. Silks, Z. Li, X. Yang, B.I. Swanson, Langmuir 16, 1793 (2000).

[3] M. Wahlgren, T. Arnebrant, Trends Biotechnol. 9, 2001 (1991).

[4] Surface and Interfacial Aspects of Biomedical Polymers, Ed. J.D. Andrade, Vol. 2, Protein Adsorption, Plenum Press, New York 1985.

[5] J.D. Andare, V. Hlady, Adv. Polym. Sci. 70, 1 (1986).

[6] B. Ivansson, I. Lundström, CDC Crit. Rev. Biocomp. 2, 1 (1986).

[7] H. Nygren, H. Arwin, S. Welin-Klinström, Colloids Surf. A Physicochem. Eng. Asp. 76, 87 (1993).

[8] H. Nygren, Biophys. J. 65, 1508 (1994).

[9] D.R. Weaver, W.G. Pitt, Biomaterials 13, 577 (1992).

[10] H.D. Jennisen, J. Chromatogr. 59, 71 (1978).

[11] I. Lundström, H. Lwing, J. Colloid Interface Sci. 136, 68 (1990).

[12] R. Azzam, N. Bashara, Ellipsometry and Polarized Light, North-Holland, Amsterdam 1977.

[13] V. Kiesel, Reflection of Light, Science Publ., Moscow 1973.

[14] D. Xu, Ph.D. Thesis, Brigham Young Univ., 2004.

[15] M. Stenberg, H. Nygren, J. Phys. C 10, 83 (1983).

[16] P. Galana, N. Noisettea, C. Estaquioa, S. Czernichow, L. Mennen, J.-C. Renversez, S. Briançon, A. Favier, S. Hercberg, Public Health Nutrition 9, 70 (2006).

[17] H. Eiskjaeligr, J.P. Bagger, C.E. Mogensen, A. Schmitz, E.B. Pedersen, Scand. J. Clinical Laboratory Investigation 52, 193 (1992). 